\title{
Low Frequency Radio Signal Polarisation Sensor with Applications in Attitude Estimation
}

\author{
Sean Maguire and Paul Robertson \\ Department of Engineering \\ University of Cambridge \\ Cambridge, UK \\ Email: \{stgm2,par10\}@cam.ac.uk
}

\begin{abstract}
A novel system for estimating the attitude (orientation) of a platform using measurements of Low Frequency (LF) radio signals is reported. The sensor system consists of an array of three orthogonal solenoid coil antennas, a three channel radio receiver circuit, a datalogging and control module and data processing algorithms. Traditional low-cost attitude estimation systems typically combine accelerometers, magnetometers and gyroscopes, but produce estimates that are sensitive to platform acceleration. This is a particular problem in highacceleration, cost-constrained environments such as small fixedwing Unmanned Aerial Vehicles. The system presented here is shown to accurately measure the axis of polarisation for signals with linear or almost linear polarisation, subject to a sign ambiguity. A representative AM radio broadcast in the LF band is shown to have a substantially linear polarisation, which is used to provide information about the sensor's attitude and shown to exhibit no drift over a period of 24 hours.
\end{abstract}

\section{INTRODUCTION}

Measurement of a system's attitude (its orientation relative to a reference coordinate frame) is essential in a range of applications, from simple smartphone tilt measurement to inertial navigation and attitude control. Within the field of attitude control, there is an increasing requirement for the use of low-cost sensors in challenging environments. For example, small low-cost Unmanned Aerial Vehicles (UAVs) are starting to be used in applications such as surveying [1], disaster relief [2], search and rescue [3] and agriculture [4]. They require an Attitude and Heading Reference System (AHRS) in order to measure and control their attitude, but this must also be small and low-cost.

In a typical low-cost AHRS, MEMS gyroscopes are used to provide an accurate high-bandwidth estimate of angular velocity, which can be integrated to produce angular position and therefore platform attitude relative to the initial orientation. In order to avoid drift, these measurements are combined with low-bandwidth vector measurements from accelerometers and often magnetometers using a filter such as the Complementary Filter or Extended Kalman Filter [5]. If it is assumed that a three-axis accelerometer measures the acceleration due to gravity and a three-axis magnetometer measures the earth's magnetic field, then two vectors can be measured with respect to the sensors that are also known with respect to the earth. The attitude of the sensors can thus be resolved as the solution of Wahba's Problem [6], [7], [8], and does not suffer from drift as the vector measurements do not drift.

Such systems have been widely used, but suffer inac- curacies in high-acceleration environments. For example in fixed-wing UAVs, where turns may cause extended centrifugal accelerations to be experienced, the accelerometer will measure the total acceleration relative to free fall and not just the acceleration due to gravity. If the accelerometer's measurements are nevertheless assumed to correspond solely to the acceleration due to gravity then large and sustained errors can be introduced into the attitude estimate. Corrections can be applied using data from additional sensors. For example GPS velocity measurements can be used to estimate and correct for the centrifugal acceleration [9]. However this can introduce significant noise, often relies on additional assumptions and has relatively low bandwidth. It also relies on an accurate GPS signal being available.

An alternative solution is to use different vectors in the attitude solution, either in addition to or instead of the acceleration vector, that are not sensitive to platform acceleration. Suitable vectors must be easily measured with small, light, low-cost and low-power equipment, while also having a known orientation. A candidate for such a vector is a unit vector defined by the polarisation of Low Frequency (LF) radio signals.

In this paper a novel sensor is presented which is capable of measuring the polarisation of LF radio signals, and which is further suitable in principal for inclusion in small UAVs due to its low size, weight and power consumption. The sensor is used to make LF polarisation measurements in order to validate the concept of attitude estimation using these vectors. In addition the polarisation properties and stability of a typical LF radio signal used for AM broadcasts are measured, showing it is suitable for this application.

\section{LOW FREQUENCY RADIO}

LF radio broadcasts are defined as having a frequency of $30-300 \mathrm{kHz}$. They are used for numerous commercial radio stations, navigation beacons and timing signals. Signals travel mainly by direct line-of-sight transmission and by groundwave (surface wave), which allows LF signals to be received over very large distances. Transmitters are typically vertical monopole antennas with a height somewhat less than a quarter of a wavelength. The vertical transmitters produce waves with substantially linear vertical polarisation of the electric field (E-field), and corresponding horizontal polarisation of the magnetic field (H-field) perpendicular to both the E-field and the direction of travel. This polarisation has been shown to be preserved by the transmission mechanisms involved (both direct and groundwave) [10], [11]. This is in contrast to other 


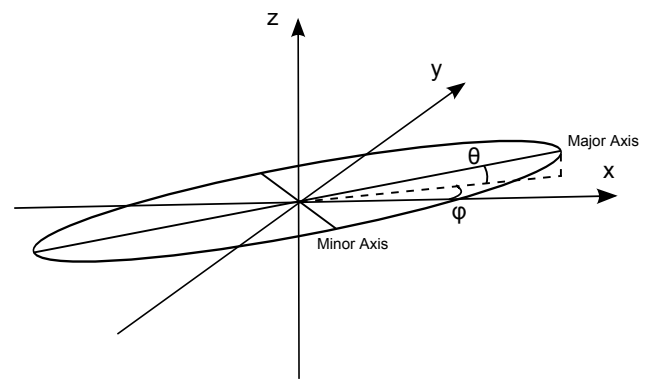

Fig. 1. Diagram of Polarisation Ellipse in 3D Coordinate System, including Definitions of Angles $\theta$ and $\phi$

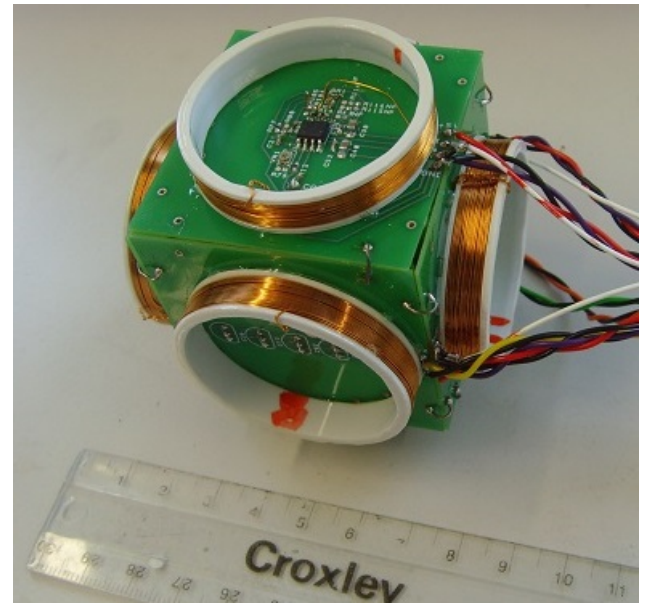

Fig. 3. Receiver Array Comprising Three Orthogonal Solenoid Antennas

mechanisms, such as skywave, which can change a signal's polarisation properties. It would thus be expected that LF signals can be received over a wide area with a substantially constant and known polarisation.

Measurements of a LF signal's H-field in three linearly independent directions allow this polarisation to be measured. In the case of substantially linear polarisation, as depicted in figure 1, this leads to a well defined vector measurement of the major axis of the polarisation ellipse, subject to a sign ambiguity. The sign ambiguity can be resolved by also taking E-field measurements, or by using an a priori estimate of receiver attitude. One or more such vectors could be used to supplement or replace the accelerometer in a traditional AHRS, thus reducing or eliminating its sensitivity to platform acceleration while potentially offering improved robustness, increased bandwidth and reduced noise compared to GPS-based methods. The process of using three-axis LF measurements to determine attitude is similar to traditional Radio Direction Finding, which uses two-axis measurements to determine location.

With reference to figure 1 , it is useful to quantify the degree of linearity of the elliptical polarisation using the ratio of the length of the major axis of the ellipse to its minor axis. The orientation of the major axis can also be defined by the angles $\theta$ and $\phi$ in a spherical coordinate system (taking $\theta$ to be measured relative to the horizontal plane for convenience).

\section{Sensor Development}

A small, light and low-cost sensor system was developed that is capable of measuring the polarisation of LF signals. It comprises a novel three-axis LF receiver, a portable datalogging and control module and signal processing algorithms which were implemented off-line. The architecture of the sensor and datalogging hardware is shown in figure 2 .

The three-axis receiver consists of an array of three orthogonal solenoid coil antennas, pictured in figure 3, used to sense the instantaneous $\mathrm{H}$-field of a LF signal in three dimensions. Each antenna is constructed from a pair of coils connected in series and mounted to opposite faces of a cube to allow a symmetrical array, minimising cross-coupling between the three axes, to be easily assembled. In addition, air-cored coils are used to reduce sensor weight. Each solenoid antenna forms a parallel resonant circuit with a set of varactors, whose capacitance can be varied by the control module. Local "selfdrive" signals generated by the control module enable the gain and phase shift of each receiver axis to be individually measured and varied. This feature was used to ensure an equal phase shift on each channel, while scale factor corrections were applied in software to account for small differences in gain. The signal from each antenna is processed by a simple radio receiver circuit including amplifiers and a bandpass filter, before being digitised by three Analogue to Digital Converters (ADCs).

A datalogging and control module was developed in order to record the LF signal detected by the three-axis receiver, in addition to controlling and characterising the receiver's frequency, phase and amplitude response. An STM32F205 32bit microcontroller was used to sample the LF signal from the receiver with its three on board simultaneous-sampling 12-bit ADCs, at sampling rates between $1 \mathrm{kSPS}$ and 1 MSPS. A high-speed SD card was used for portable data storage.

The raw data stored by the datalogging and control module was subsequently processed off-line in order to estimate the polarisation of the LF signal. First the signal was digitally filtered using an elliptic filter with a bandwidth of $100 \mathrm{~Hz}$ to isolate the frequency of interest, before estimates of the major and minor axes of the polarisation ellipse of the resulting signal were formed using Singular Value Decomposition. This technique has been used to extract the polarisation ellipse in other applications [12]. Output rates of between $1 \mathrm{~Hz}$ and 10 $\mathrm{Hz}$ were used for the polarisation vector estimates, although higher rates are possible in principle.

\section{EXPERIMENTAL RESULTS}

The sensor was first tested using locally-generated signals in order to verify its operation. A ferrite-cored solenoid coil antenna driven from a signal generator was used to transmit a $198 \mathrm{kHz} \mathrm{H}$-field signal with linear polarisation. The transmit antenna and the receiver under test were placed in an RF screened room in order to eliminate external signals, and the signal measured by the receiver was recorded with the transmitting antenna in different orientations at a range of 50 $\mathrm{cm}$. Figure 4 shows the measured angle of the major axis of the $\mathrm{H}$-field polarisation ellipse as the transmitting antenna is rotated through $90^{\circ}$ in the horizontal plane. Agreement between the actual and measured angle is within approximately 


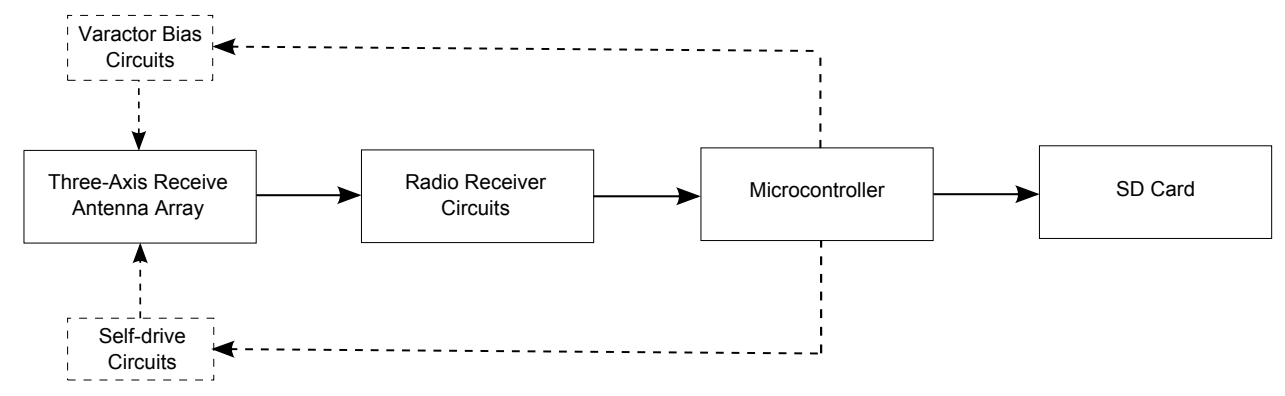

Fig. 2. Block Diagram of Receiver Including Solenoid Antenna Array and Processing and Control Element, Showing Main Signal Path (Solid Lines) and Control/Characterisation Circuitry (Dashed Lines)

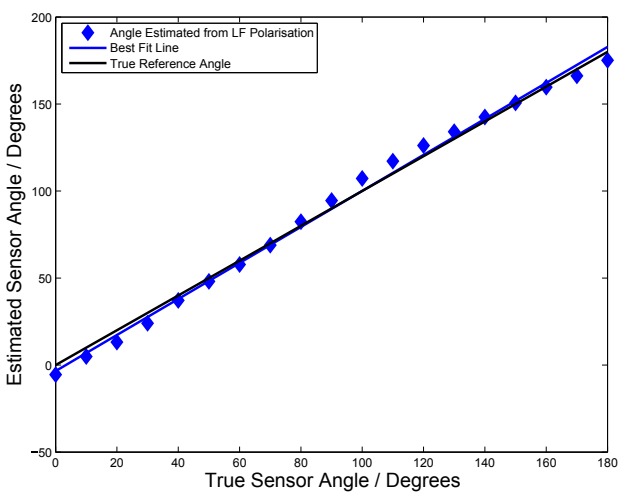

Fig. 4. Sensor Angle Inferred from Locally Generated $198 \mathrm{kHz}$ Signal, Plotted Alongside True Angle Reference, for Sensor Angles Between $0^{\circ}$ and $180^{\circ}$ in the Horizontal Plane

$7^{\circ}$. This error is likely due to both cross-coupling of the signal between the three sensor axes and signal interaction with the screened room.

Cross-coupling between the three receiver axes was measured and coupled signal magnitudes of up to $8 \%$ of the original signal were observed, leading to angle errors of up to $6.5^{\circ}$. The coupling also exhibits a phase shift, and can therefore introduce small errors to the measured ratio of the polarisation major and minor ellipse values. Measurements of the coupling amplitude and phase shift offer the potential for additional calibration to improve the accuracy of the sensor.

The sensor was subsequently used to measure an LF signal used for AM broadcasts. BBC Radio 4 longwave, transmitted from Droitwich on $198 \mathrm{kHz}$, was selected. Measurements were made in Cambridge, at a range of approximately 150 $\mathrm{km}$ from the transmitter. As expected, the signal was found to be highly polarised. Figure 5 shows an example of an observed polarisation ellipse, formed by plotting instantaneous measurements of the LF signal sampled at 1 MSPS for a duration of $200 \mu \mathrm{s}$. The ratio of the major axis to the minor axis of the ellipse was generally found to be in the range $8-10$, indicating almost linear polarisation. The ellipse was further found to be oriented in the expected direction, with the major axis close to the horizontal plane $\left(\theta<5^{\circ}\right)$ and close to the perpendicular to the line between transmitter and receiver $\left(\phi<7^{\circ}\right)$, where angles are defined with reference to figure $1, z$ is the vertical and $y$ is the direction of travel of the LF wave. These errors are within the range expected due to cross-coupling. However, significant distortions to the

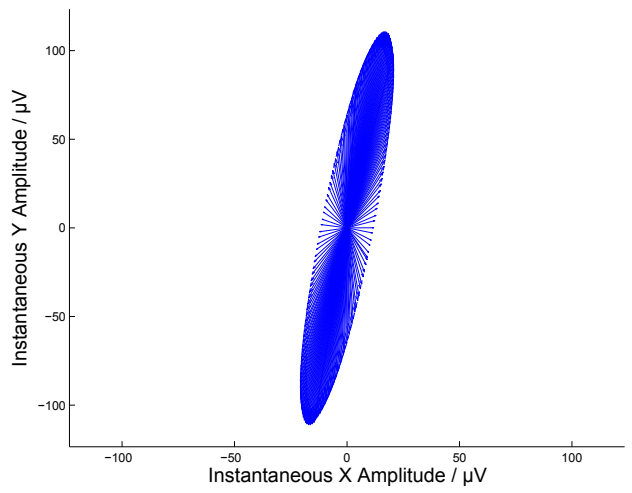

Fig. 5. Typical 2D Polarisation Ellipse of Radio 4 Longwave Signal, Formed as a Vector Plot of the Instantaneous Voltages on two Receiver Axes, Sampled at 1 MSPS over $200 \mu \mathrm{s}$

polarisation were observed when taking measurements near to metal structures or very close to the ground.

In order to verify that this signal can be used to estimate the receiver's attitude, the receiver was placed in a horizontal position and rotated in the horizontal plane by known angles while measuring the polarisation of the $198 \mathrm{kHz}$ Radio 4 carrier signal. As shown in figure 6 it was found that the 2D orientation of the receiver could be inferred (with a sign ambiguity) to within $6^{\circ}$ using only measurements of $\phi$. These errors are consistent with those observed due to cross-coupling between the receiver axes, suggesting they could be reduced by additional calibration. In order to perform full 3D attitude estimation, two or more vectors would be necessary (derived from additional LF measurements or other sensors such as accelerometers and magnetometers).

In order to quantify its stability the selected LF signal was measured over a 24 hour period, sampled at $1.12 \mathrm{kSPS}$, and estimates of the orientation of the major axis of the polarisation ellipse were calculated at one second intervals (subject to the sign ambiguity). Figure 7 shows a histogram of measurements of $\theta$ over the 24 hour period and figure 8 shows measurements of $\phi$ over the same period. As summarised in table I, the signal properties were found to differ substantially between night $(8 \mathrm{pm}$ to $6 \mathrm{am})$ and day. Although the measurements in both cases have similar means (with only a $2.6 \%$ change in the mean value of $\theta$ and $1.8 \%$ change in the mean value of $\phi$ going from day to night), the variance is significantly higher at night. This is believed to be the result of changes in the RF propagation environment. In particular, it is well known that 


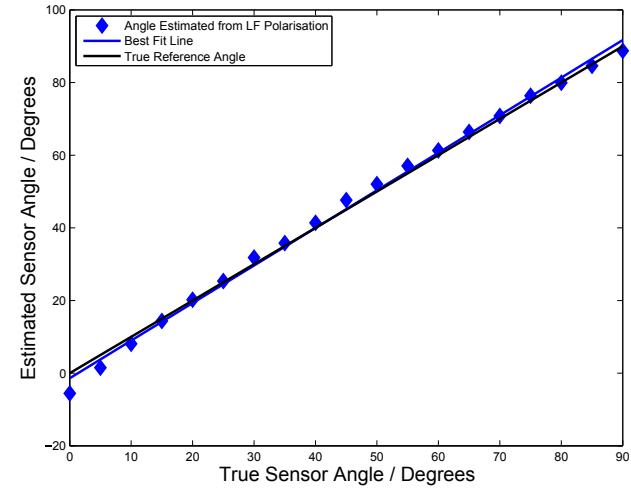

Fig. 6. Sensor Angle Inferred from Measurements of Radio $4198 \mathrm{kHz}$ Carrier Wave, Plotted Alongside True Angle Reference, for Sensor Angles Between $0^{\circ}$ and $90^{\circ}$ in the Horizontal Plane

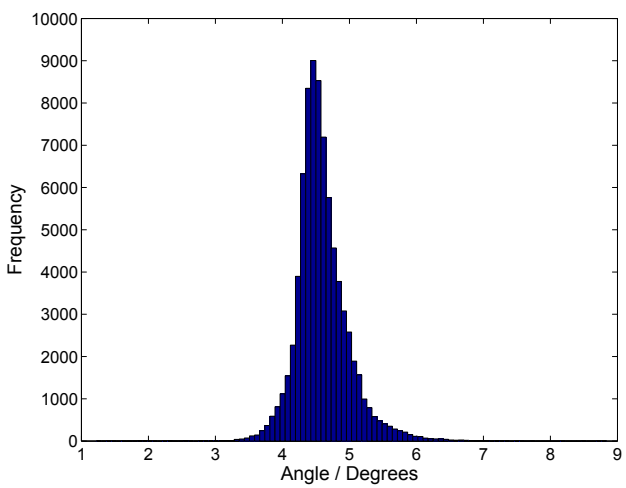

Fig. 7. Angle $\theta$ Between Estimated Primary Axis of Polarisation Ellipse and Horizontal Plane, Measured Over 24 Hours

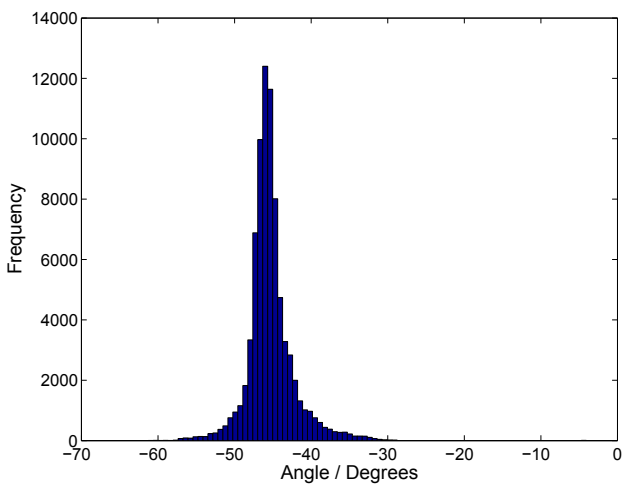

Fig. 8. Angle $\phi$ of Estimated Primary Axis of Polarisation Ellipse Within the Horizontal Plane, Measured Over 24 Hours

TABLE I. Mean and Standard Deviation of Measured Angles

\begin{tabular}{|c|c|c|}
\hline & Day (6am-8pm) & Night (8pm-6am) \\
\hline$\theta$ & $\mu=4.5^{\circ}, \sigma=0.3^{\circ}$ & $\mu=4.7^{\circ}, \sigma=0.5^{\circ}$ \\
\hline$\phi$ & $\mu=-45.7^{\circ}, \sigma=1.5^{\circ}$ & $\mu=-44.8^{\circ}, \sigma=4.3^{\circ}$ \\
\hline
\end{tabular}

skywave propagation is more significant at night. In addition to affecting the propagation of the LF signal of interest, there are other European stations broadcasting on the same frequency which are likely to be more strongly received at long range by night, and thus cause greater interference. This increase in variance would reduce the useful sensor bandwidth at night.

\section{CONCLUSION}

A novel three-axis LF radio polarisation sensor has been demonstrated and shown to accurately measure the major axis of polarisation for LF signals. The sensor has been used to measure the polarisation of a widely available LF radio signal, and the observed polarisation was found to agree closely with that predicted by theory. The major axis of the polarisation of this signal has been shown to determine the 2D orientation of the sensor, subject to resolution of the sign ambiguity. The orientation of the major axis of the polarisation ellipse was found to be stable over time, although with a higher variance at night. These results support the assertion that such a sensor could be used to supplement or replace the accelerometer in an AHRS in some applications, in order to reduce its sensitivity to acceleration and increase its robustness.

Further work is needed to reduce sensor errors through additional calibration, validate the sensor in a greater range of geographical locations and integrate it into an AHRS providing full 3D attitude solutions.

\section{REFERENCES}

[1] S. Siebert and J. Teizer, "Mobile 3d mapping for surveying earthwork projects using an unmanned aerial vehicle (uav) system," Automation in Construction, vol. 41, no. 0, pp. 1 - 14, 2014. [Online]. Available: http://www.sciencedirect.com/science/article/pii/S0926580514000193

[2] H. Bendea, P. Boccardo, S. Dequal, F. Giulio-Tonolo, D. Marenchino, and M. Piras, "Low cost UAV for post-disaster assessment," vol. 37, no. B8, pp. 1373-1379, 2008.

[3] M. A. Goodrich, B. S. Morse, D. Gerhardt, J. L. Cooper, M. Quigley, J. A. Adams, and C. Humphrey, "Supporting wilderness search and rescue using a camera-equipped mini uav," Journal of Field Robotics, vol. 25, no. 1-2, pp. 89-110, 2008. [Online]. Available: http://dx.doi.org/10.1002/rob.20226

[4] L. S. J Berni, P J Zarco-Tejada and E. Fereres, "Thermal and narrowband multispectral remote sensing for vegetation monitoring from an unmanned aerial vehicle," Geoscience and Remote Sensing, IEEE Transactions on, vol. 47, no. 3, pp. 722 -738, March 2009.

[5] D. Gebre-Egziabher, R. C. Hayward, and J. D. Powell, "Design of multi-sensor attitude determination systems," IEEE Transactions on Aerospace and Electronic Systems, vol. 40, no. 2, pp. 627-649, April 2004.

[6] J. L. Farrell, J. C. Stuelpnagel, R. H. Wessner, and J. R. Velman, "A least squares estimate of satellite attitude," SIAM Review, vol. 8, no. 3, pp. 384-386, 1966.

[7] M. D. Shuster and S. D. Oh, "3-axis attitude determination from vector observations," Journal of Guidance and Control, vol. 4, no. 1, pp. 7077, January-February 1981.

[8] F. L. Markley and D. Mortari, "Quaternion attitude estimation using vector observations," Journal of the Astronautical Sciences, vol. 48, no. 2-3, pp. 359-380, April-September 2000.

[9] M. Euston, P. Coote, R. Mahony, J. Kim, and T. Hamel, "A complementary filter for attitude estimation of a fixed-wing UAV," in Proceedings of the 2008 IEEE/RSJ International Conference on Robots and Intelligent Systems, Nice, France, 2008, Proceedings Paper, pp. 340-345.

[10] K. A. Norton, "The physical reality of space and surface waves in the radiation field of radio antennas," Proceedings of the Institude of Radio Engineers, vol. 25, no. 9, pp. 1192-1202, September 1937.

[11] R. W. P. King and S. S. Sandler, "The electromagnetic field of a vertical electric dipole over the earth or sea," IEEE Transactions on Antennas and Propagation, vol. 42, no. 3, pp. 382-389, March 1994.

[12] R. de Franco and G. Musacchio, "Polarization filter with singular value decomposition," Geophysics, vol. 66, no. 3, pp. 932-938, 2001. [Online]. Available: http://geophysics.geoscienceworld.org/content/66/3/932.abstract 